\title{
Ensuring tightness of metal-polymer joints of hydropneumatic systems
}

\author{
P. Ogar, D. Gorokhov, L. Mamaev, V. Fedorov \\ Mechanical Department \\ Bratsk State University \\ Bratsk, Russia \\ ogar@brstu.ru
}

\begin{abstract}
The issues of ensuring of guaranteed, with a given probability of the flow of the medium, the tightness of metalpolymeric sealing joints of the hydraulic systems of apparatuses and units are considered. Taking into account the mutual influence of the microasperities of the rough surface, the dependences of the relative contact area, the gap density in the joint and the probability of the medium flowing from the dimensionless force elastic geometric parameter $f_{q}$ are determined. These contact characteristics determine the functional of the permeability $C_{u}$ which characterizes the sealing capacity of the sealing joint. Their role in various periods of loading of a sealing joint by a dimensionless load is shown. From the dependence of the functional of the permittivity $C_{u}$ on the dimensionless force elastic geometric parameter $f_{q}$, it is possible to distinguish three zones of sealing: initial, due to the indentation of the highest asperities; stable, by reducing the gap density; effective, due to the fusion of individual contact spots. It is shown that due to the fusion of single contact spots at $f_{q}>f_{q e}$, the probability of the medium flowing sharply decreases. For the probability value $v_{k}=0.0001$, the values $f_{q e}=0.50 \ldots 0.60$ which corresponds to the relative contact area $\eta=0.58 \ldots 0.63$. This result agrees well with the value of the relative actual contact area obtained using percolation theory, in which a continuous closed cluster is formed, i.e. overlapping of all microchannels in the joint is ensured.
\end{abstract}

Keywords - tightness; metal-polymer joins; relative contact area; gap density in the joint; probability of a medium flowing

\section{INTRODUCTION}

Sealing joints are widely used in pipeline communications of hydro-pneumatic systems of aircraft, as well as in engines, for hermetic sealing of cabins. Hydropneumatic systems of aircraft can be divided into liquid (hydraulic, fuel, oil, antidestruction, etc.) and gas (pneumatic, oxygen, fire, heating, conditioning, etc.). The share of hydropneumatic fuel aggregates in aircraft equipment can be up to $25 \%$ in terms of nomenclature and $35 \%$ in metal consumption. Their reliability is largely determined by the quality of the sealing joints. Ensuring the reliability of sealing joints is laid at the design stage.

The complexity of modern power control systems can be judged by the example of the hydraulic system of an airplane of the type XV-70 [1], which includes 12 pumps, 129 actuators, 170 valves. The total volume of the hydraulic system is 830 liters, the length of pipeline communications is more than $1600 \mathrm{~m}$. The system has 3900 sealing joints. In recent decades, the number of powertrain units has tripled, the length of the pipelines was $2.5 \ldots 3.5 \mathrm{~km}$. There are hydraulic systems at 48 and $70 \mathrm{MPa}$.

The tightness of the sealing joints is provided by loading them with a compressive force (contact sealing pressures) and is largely determined by the stress-strain state in the contact zone and depends on the contact interaction of the rough surfaces. The main contact characteristics ensuring the tightness of the sealing joints are the relative contact area, the density of the gaps in the joint, the degree of fusion of contact spots of single asperities, which determines the probability of the medium flowing [2]

Widely used in sealing technology are low-modular polymer materials in the form of coatings or individual parts [3]. According to the strength criteria, structural materials are low modulus with elastic modulus values of $E<10^{3} \mathrm{MPa}$.

To quantify the leak tightness, use the leakage, that means rate-the mass or volume of the medium per unit time. To evaluate the sealing capacity of the sealing joint, the nondimensional permeability functional is used

$$
C_{u}=\frac{\Lambda^{3} v_{k}}{4(1-\eta)^{2}},
$$

where is the density of gaps in the joint; $\eta$ is the relative contact area; $v_{k}$ is the the probability of a medium flowing which depends on the fusion of individual contact spots.

All the parameters entering into Eq. (1) depend on the parameters of microgeometry and the dimensionless force elastogeometric parameter $f_{q}$.

The purpose of this paper is to determine the role of the parameters included in Eq. (1) in ensuring tightness of joints and to determine the conditions for ensuring a guaranteed tightness, taking into account the mutual influence of microasperities. The guaranteed tightness corresponds to the set value of the probability of the flow of the medium $v_{k}$, for example $v_{k}=0.0001$. 


\section{MODEL OF A ROUGH SURFACE}

At present, models of roughness and the theory of contacting rough surfaces developed by GreenwoodWilliamson, N.B. Demkin and their followers [4, 5] are used to solve the problems of tribology. However, the use of such models for solving the problems of ensuring the tightness of connections with low-modulus materials leads to significant errors which is explained by the following: 1) the contact pressure of the sealing is about 1-2 orders of magnitude higher than that of friction, and the mutual influence of the contacting asperities must be taken into account; 2) in the sealing joint, it is possible to contact all the asperities which requires the description of the whole profile bearing curve and not only its initial part; 3) in determining the gap density, displacements of the points of the surface of the asperities are not taken into account; 4) it does not take into account the fusion of single contact spots.

Therefore, we use the discrete roughness model given in [1]. Microasperities can be represented as a set of identical spherical segments with radius, base and height. The height distribution of the asperities corresponds to the bearing curve of the real surface profile which is described by the regularized beta function:

$$
\eta(\varepsilon)=\frac{\mathrm{B}_{\varepsilon}(p, q)}{\mathrm{B}(p, q)},
$$

where, $\mathrm{B}_{\varepsilon}(p, q), \mathrm{B}(p, q)$ are respectively, the incomplete and complete beta-functions;

$$
p=\left(\frac{R_{p}}{R_{q}}\right)^{2}\left(\frac{R_{\max }-R_{p}}{R_{\max }}\right)-\frac{R_{p}}{R_{\max }}, q=p\left(\frac{R_{\max }}{R_{p}}-1\right) ;
$$

$R_{p}, R_{q}, R_{\mathrm{max}}$ are the height parameters of roughness.

The density of the height distribution function of the asperities is described by the expression:

$$
\varphi_{n}^{\prime}(u)=\frac{u^{p-2}(1-u)^{q-2}[(p-1)(1-u)-(q-1) u]}{\varepsilon_{s}^{p-1}\left(1-\varepsilon_{s}\right)^{q-1}},
$$

where $u$ is the distance from the peak level to the top of the asperities; $\varepsilon_{S}$ is determined from the condition $\varphi_{n}\left(\varepsilon_{S}\right)=1$; $\omega=1-\varepsilon_{s}$.

Radius of the spherical segment is

$$
r=a_{c}^{2} /\left(2 \omega R_{\max }\right) .
$$

Fig. 1 shows the density distribution of the height asperities for the examples of the relative contact area below.

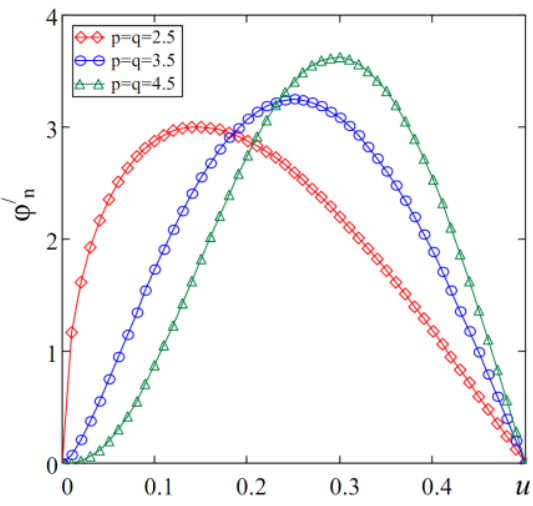

b)

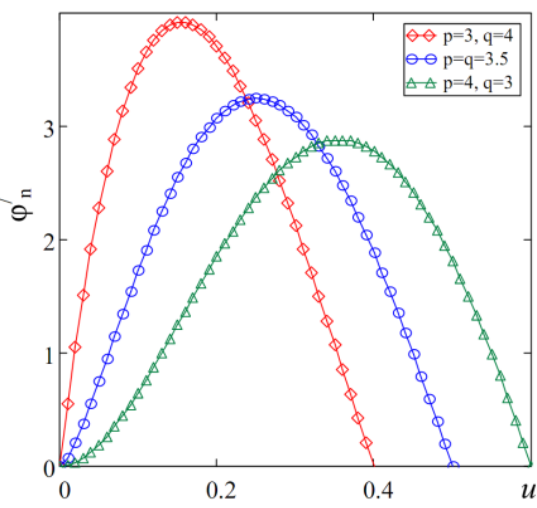

Fig. 1. Density distribution of asperities for different values of $p$ and $q$.

As it follows from Fig. 1, the variation of the parameters $p$ and $q$ let to effectively control the density distribution of the asperities in the height of the rough layer.

\section{CONTACT OF A RIGID ROUGH SURFACE WITH AN ELASTIC HALF-SPACE}

\section{A. Relative contact area}

As follows from [5-7], and also from the recent publication of the authors [1], in determining the relative contact area, especially with the use of low-modulus materials, the mutual influence of microasperities should be taken into account. Below we give a system of equations [1], the solution of which will allow us to determine the dependence of the relative contact area $\eta$ on the dimensionless force elastic geometric parameter $f_{y}$, which is determined by equation

$$
f_{q}=\frac{q_{c} a_{c}}{E^{*} \omega R_{\max }}
$$

where $E^{*}$ is the reduced modulus of elasticity.

To contact a rough surface with an elastic half-space, we have

$$
f_{q}(\varepsilon)=\frac{q_{c}(\varepsilon) a_{c}}{E^{*} \omega R_{\max }}=\frac{\frac{8}{3 \pi} \int_{0}^{\min \left(\varepsilon, \varepsilon_{s}\right)} \eta_{i}^{1,5} \varphi_{n}^{\prime}(u) d u}{1-\int_{0}^{\min \left(\varepsilon, \varepsilon_{s}\right)} \psi_{\eta}\left(\eta_{i}\right) \varphi_{n}^{\prime}(u) d u},
$$


where $\psi_{\eta}\left(\eta_{i}\right)=\frac{2}{\pi}\left[\arcsin \eta_{i}^{0.5}-\sqrt{\eta_{i}\left(1-\eta_{i}\right)}\right]$.

Relative contact area is

$$
\eta(\varepsilon)=\int_{0}^{\min \left(\varepsilon, \varepsilon_{s}\right)} \eta_{i} \varphi_{n}^{\prime}(u) d u .
$$

To determine the dependence $\eta\left(f_{q}\right)$, it is necessary to exclude the parameter from the dependences $f_{q}(\varepsilon)$ and $\eta(\varepsilon)$. The dependences $\eta\left(f_{q}\right)$ for the distribution densities of the asperities corresponding to Fig. 1 are shown in Fig. 2 and are denoted by dots. The line in Fig. 2a is indicated an analogous relationship without taking into account the mutual influence of the asperities, from which it follows that when $\eta=1$ using a discrete roughness model, the case is possible. This contradicts the data of papers [8-12] devoted to the study of almost complete contact between rough surfaces and confirms the correctness of the chosen approach.
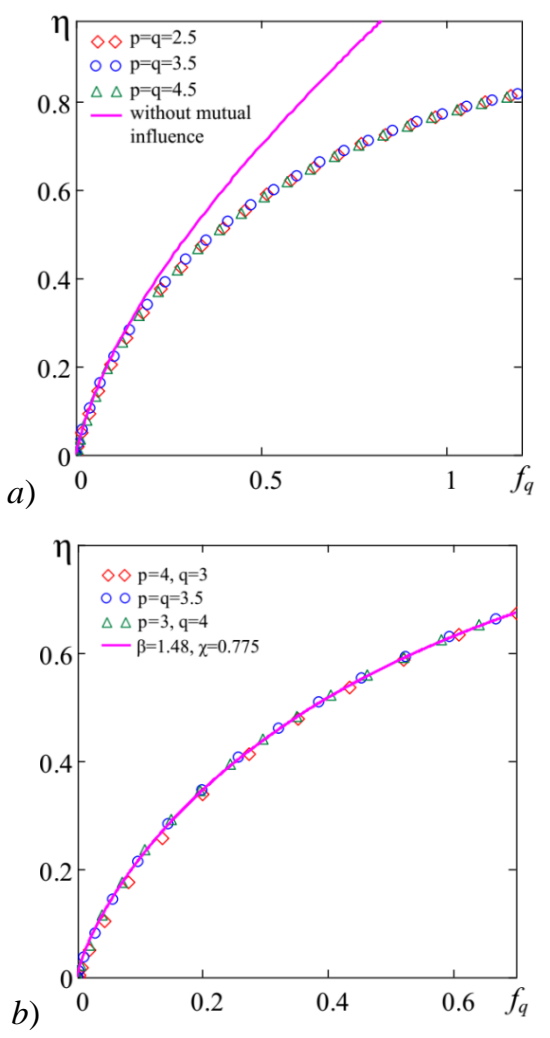

Fig. 2. Dependence of the relative contact area on the dimensionless parameter $f_{q} \quad$ a) taking into account (point) and without taking into account (the line) the mutual influence of asperities; b) for different values of the parameters $p$ and $q$.

It follows from Fig. 2, the dependences for different values of the parameters $\mathrm{p}$ and $\mathrm{q}$ practically merge into one line.
Therefore, for engineering calculations it is expedient to describe them by a single equation

$$
\eta=1-\exp \left(-1,48 f_{q}^{0,775}\right) \text {. }
$$

The approximation of the analytical dependencies $\eta\left(f_{q}\right)$ describes them quite accurately, and therefore can be recommended for engineering calculations of the relative area under elastic contact.

\section{B. Density of gaps in the joint}

To determine the volume of the intercontact space, it is necessary to determine the volumes of gaps attributable to single contacting and noncontacting asperities $[1,13]$

$$
V_{i}=\left\{\begin{array}{c}
V_{r i}=2 \pi \int_{a_{r i}}^{a_{c}}\left[z_{20}(\rho)-z_{10}(\rho)\right] \rho d \rho \\
V_{0 i}=2 \pi \int_{0}^{a_{c i}}\left[z_{2 r}(\rho)-z_{1 r}(\rho)\right] \rho d \rho,
\end{array}\right.
$$

where $z_{10}, z_{20}$ are the equations describing surfaces of noncontacting asperities and half-spaces; $z_{1 r}, z_{2 r}$ are the equations describing the surfaces of contacting asperities and half-spaces.

Then the total volume of the intercontact space at the joint is described by equation

$$
V_{c}=\sum_{i=1}^{n_{r}} V_{r i}+\sum_{i=1}^{n_{c}-n_{r}} V_{0 i},
$$

and the corresponding gap density is equal to

$$
\Lambda(\varepsilon)=\frac{1}{A_{c i} R_{\max }}\left[\int_{0}^{\min \left(\varepsilon, \varepsilon_{S}\right)} V_{r i} \varphi_{n}^{\prime}(u) d u+\int_{\min \left(\varepsilon, \varepsilon_{S}\right)}^{\varepsilon_{S}} V_{V_{i}} \varphi_{n}^{\prime}(u) d u\right] .
$$

Taking into account that $\Lambda_{r i}=V_{r i} /\left(A_{c i} R_{\max }\right)$ $\Lambda_{0 i}=V_{0 i} /\left(A_{c i} R_{\max }\right)$, the Eq. (12) can be represented in the form

$$
\Lambda(\varepsilon)=\int_{0}^{\min \left(\varepsilon, \varepsilon_{S}\right)} \Lambda_{r i} \varphi_{n}^{\prime}(u) d u+\int_{\min \left(\varepsilon, \varepsilon_{S}\right)}^{\varepsilon_{S}} \Lambda_{0 i} \varphi_{n}^{\prime}(u) d u .
$$

To determine the volume of the intercontact space, it is necessary to determine the volumes of gaps attributable to single contacting and noncontacting asperities $[1,13]$

$$
V_{i}=\left\{\begin{array}{c}
V_{r i}=2 \pi \int_{c}^{a_{c}}\left[z_{20}(\rho)-z_{10}(\rho)\right] \rho d \rho \\
a_{r i} \\
V_{0 i}=2 \pi \int_{0}^{a_{c i}}\left[z_{2 r}(\rho)-z_{1 r}(\rho)\right] \rho d \rho,
\end{array}\right.
$$


where $z_{10}, z_{20}$ are the equations describing surfaces of noncontacting asperities and half-spaces; $z_{1 r}, z_{2 r}$ are the equations describing the surfaces of contacting asperities and half-spaces.

Then the total volume of the intercontact space at the joint is described by equation

$$
V_{c}=\sum_{i=1}^{n_{r}} V_{r i}+\sum_{i=1}^{n_{c}-n_{r}} V_{0 i}
$$

and the corresponding gap density is equal to

$$
\Lambda(\varepsilon)=\frac{1}{A_{c i} R_{\max }}\left[\int_{0}^{\min \left(\varepsilon, \varepsilon_{S}\right)} V_{r i} \varphi_{n}^{\prime}(u) d u+\int_{\min \left(\varepsilon, \varepsilon_{S}\right)}^{\varepsilon_{S}} V_{0 i} \varphi_{n}^{\prime}(u) d u\right]
$$

Taking into account that $\Lambda_{r i}=V_{r i} /\left(A_{c i} R_{\max }\right) \quad$ и $\Lambda_{0 i}=V_{0 i} /\left(A_{c i} R_{\max }\right)$, the Eq. (12) can be represented in the form

$$
\Lambda(\varepsilon)=\int_{0}^{\min \left(\varepsilon, \varepsilon_{S}\right)} \Lambda_{r i} \varphi_{n}^{\prime}(u) d u+\int_{\min \left(\varepsilon, \varepsilon_{S}\right)}^{\varepsilon_{S}} \Lambda_{0 i} \varphi_{n}^{\prime}(u) d u .
$$

Expressions for $\Lambda_{r i}=\Lambda_{r i}(\varepsilon, u)$ and $\Lambda_{r i}=\Lambda_{r i}(\varepsilon, u)$ are given in [1].

a)
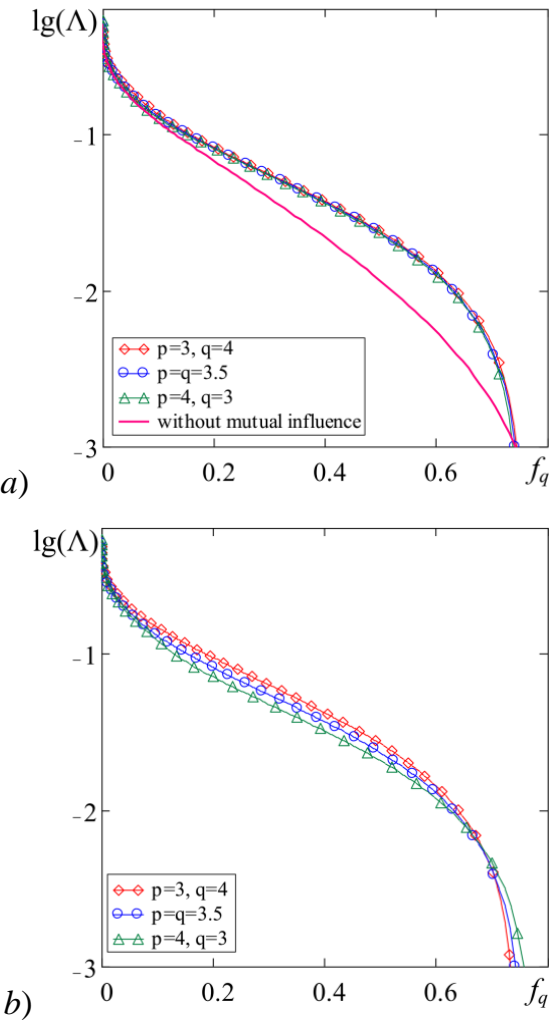

Fig. 3. Density of gaps in the joint: with taking into account and without taking into account the mutual influence of asperities (a); for different values of $p$ and $q(b)$
In Fig. 3 shows the dependence of the gap density $\Lambda$ on the complex dimensionless force elastic geometric parameter $f_{q}$. From Fig. 3 it follows that $\Lambda$ it depends on the influence conditions of the asperities and to a much lesser extent depends on the values of the parameters $p$ and $q$.

\section{Probability of a medium flowing}

The probability of a medium flowing is determined by the degree of confluence of single contact spots. In [1], a model of a single asperity was used for this (Fig. 4), which is described by equation

$$
z(x, y)=v+\omega[f(x)+f(y)]
$$

where $\quad x=\frac{2 X}{S_{m x}}, \quad y=\frac{2 Y}{S_{m y}}, \quad z=\frac{Z}{R_{\max }}$,

$$
S_{m x}=\sqrt{A_{c i} K_{s}}, \quad S_{m y}=\sqrt{A_{c i} / K_{s}}, \quad K_{s}=S_{m x} / S_{m y},
$$$$
f(t)= \begin{cases}0,5-t, & |t| \leq 0,5, \\ (1-|t|)^{2}, & 0,5 \leq|t| \leq 1 .\end{cases}
$$

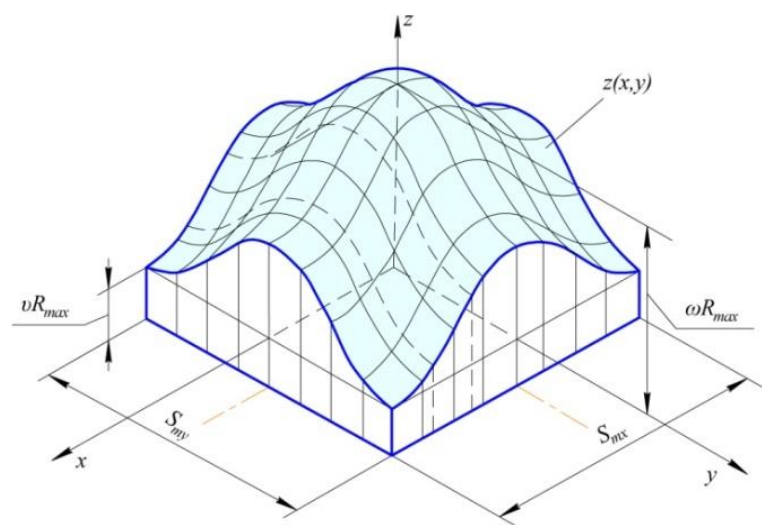

Fig. 4. Model of a single asperity

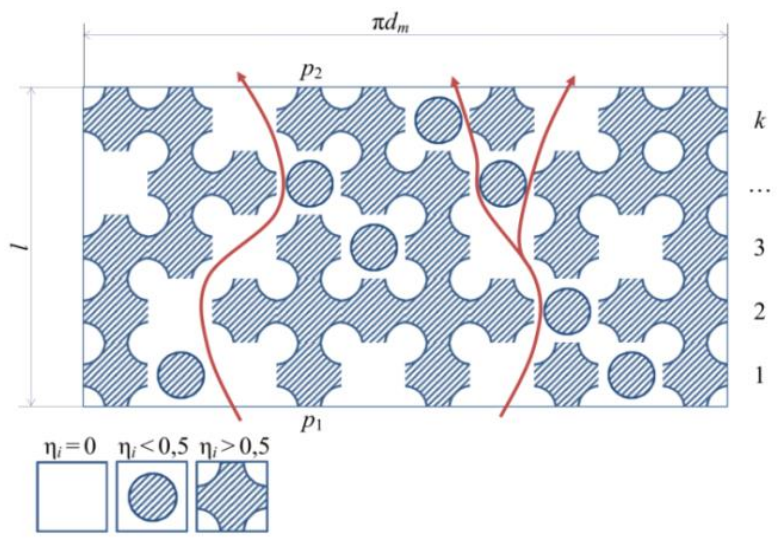

Fig. 5. Scheme of the sealing joint 
For this asperity, the distribution of material along the height $\omega R_{\max }$ is linear, i.e. similarly as for a spherical segment at $r \gg R_{\max }$. The cross-section of the asperity at the level $v+0.5 \omega$ concerns the contour of the asperity. If two adjacent asperities occur $\eta_{i}>\eta_{i}^{*}$, they will merge.

Suppose that a flat sealing joint of a length $\pi d_{m}$ and a width $l$ consists $k$ of rows of asperities (Fig. 5). The probability of a medium flowing [1] is

$$
v_{k}(\varepsilon)= \begin{cases}\left(1-x^{*}\right), & x^{*}<\frac{1}{3} \\ 3^{k-1}\left(1-x^{*}\right) k & x^{*} \geq \frac{1}{3}\end{cases}
$$

where $k$ is the number of rows of microasperities along the width of the sealing zone;

$$
\begin{array}{r}
x^{*}(\varepsilon)=\int_{0}^{\varepsilon} x_{i}^{*}(\varepsilon, u) \varphi^{\prime}(u) d u ; \\
x_{i}^{*}(\varepsilon, u)= \begin{cases}0, & \eta_{i}(\varepsilon, u)<\eta_{i}^{*} ; \\
1, & \eta_{i}(\varepsilon, u) \geq \eta_{i}^{*} ;\end{cases}
\end{array}
$$

$\eta_{i}^{*}=0,5 \ldots 0,52$ is critical value $\eta_{i}$.

The dependencies $v_{k}\left(f_{q}\right)$ are shown in Fig. 6.

a)

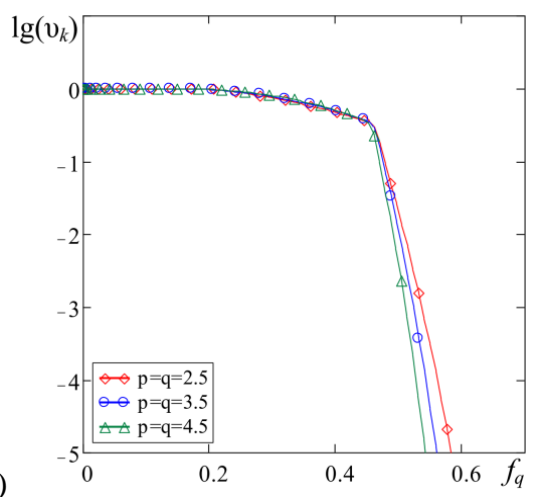

b)

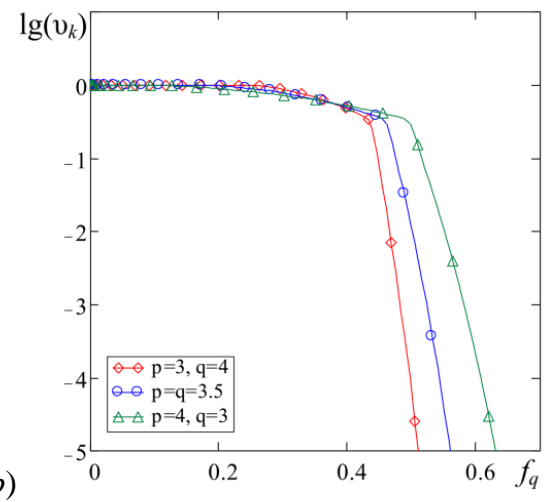

Fig. 6. The dependence of the probability of the flow of the medium $v_{k}$ from the dimensionless parameter $f_{q}$
It follows from Fig. 6, because of the fusion of single contact spots at $f_{q}>f_{q e}$, the probability of the medium flowing sharply decreases.

\section{RESULTS AND DISCUSSION}

Substituting the Eqs. (10), (13) and (15) in (1), we obtain the dependence of the permeability functional $C_{u}$ on the dimensionless force elastic geometric parameter $f_{q}$.

The mass or volume flow of the medium is determined by the equations

$$
\begin{gathered}
G=C_{f} \cdot C_{u}, \quad Q=C_{g} \cdot C_{u} ; \\
C_{f}=\frac{\pi d_{m} R_{\max }^{3} \rho \Delta p}{2 l \mu}, \quad C_{g}=\frac{\pi d_{m} R_{\max }^{3}\left(p_{1}^{2}-p_{2}^{2}\right)}{4 l \mu} ;
\end{gathered}
$$

where $\rho$ is the density of the medium to be sealed; $p_{1}$ and $p_{2}$ are inlet and outlet pressures; ; $\mu$ is dynamic viscosity; $l$ is width of the sealing zone.

When designing the sealing joint, it is convenient to determine the required permeability function $C_{u}$ from the specified $\rho, p_{1}, p_{2}, \mu$ and assigned $d_{m}, l$ and $R_{\max }$ taking into account $G$ (or $Q$ ) from equations (16) and (17)

$$
C_{u}=\frac{2 l \mu G}{\pi d_{m} R_{\max }^{3} \rho \Delta p},
$$

and then from the dependencies $C_{u}\left(f_{q}\right)$ determine the power parameter $f_{q}$ that provides the given level $C_{u}^{*}$, and therefore, the specified leakage value $G$.

The dependencies are shown in Fig. 7. As it follows from Fig. 7, there are three zones of sealing: a) initial $\left(f_{q} \leq 0.05\right)$, due to the introduction of the highest asperities; b) stable $\left(0.05 \leq f_{q} \leq f_{q e}\right)$, by reducing the gap density; c) effective $\left(f_{q}>\right.$ $f_{q e}$, due to the fusion of individual contact spots.

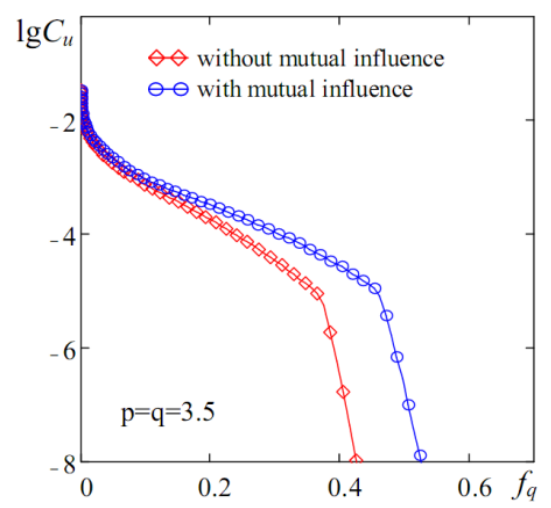

Fig. 7. Dependence of the functional of the permeability of $C_{u}$ on of the forse elastic geometric parameter $f_{q}$ 
The value of $f_{q e}$ is characterized by the onset of a sharp decrease in the permeability functional (Fig. 7) due to a decrease in the probability of medium permeation (Fig. 6). For a given level of probability of the flow of the medium, for example $v_{k}=0.0001$, the value $f_{q e}=0.50 \ldots 0.60$. With taking into account Eq. (10), this occurs at a relative contact area $\eta=0.58 \ldots 0.63$, for $p=q=3.5$, we have $\eta=0.60$. This result agrees well with the data of [14] obtained using percolation theory (permeation), which determine the value of the relative actual contact area at which a continuous closed cluster is formed, i.e. overlapping of all microchannels in the joint is ensured.

\section{CONCLUSION}

1. The main contact characteristics that determine the sealing capacity of compounds are the relative contact area, the gap density in the joint and the degree of fusion of single contact spots.

2. The density of gaps in the joint is determined taking into account the mutual influence of asperities, the value of which gives a margin for tightness in comparison with the values obtained without taking into account the mutual influence.

3. The probability of a medium flowing is determined by the degree of confluence of single contact spots and the width of the sealing zone. The fusion of contact spots occurs $\eta_{i}>\eta_{i}^{*}$ for two adjacent asperities.

4. The guaranteed tightness depends on the distribution of asperities along the height of the rough layer and is provided with the relative contact area in the joint $\eta=0.58 \ldots 0.63$. This result is in good agreement with the data obtained using the percolation theory.

\section{References}

[1] N.M. Belyaev, E.I. Uvarov, and Yu.M. Stepanchuk, Pneumohydraulic systems. Moskov. 1988, $271 \mathrm{p}$.

[2] P. Ogar, S. Belokobylsky, and D. Gorokhov, "Contact mechanics of rough surfaces in hermetic sealing study", chapter in: Contact and Fracture Mechanics, Pranav H. Darji, ed. 2018, in press.

[3] Seals and sealing equipment. Handbook of 2nd ed. A.I. Golubev and L.A. Kondakov, Eds. Moscow: Mechanical Engineering, 1994, 445 p.

[4] J.A. Greenwood and J.B.R. Williamson, " Contact of nominally flat sufaces”, Proc. Roy.Soc. 1966. V. A295. pp. 301-313.

[5] N.B. Demkin, Contact of rough surfaces, Moscow: Nauka. 1970, 227 p

[6] I.G. Goryacheva and N.M. Dobychin, Contact problems in tribology. Moscow: Mechanical Engineering, 1988, 256 p.

[7] A.P. Makushkin, Polymers in friction and seal assemblies at low temperatures. Moscow: Mechanical Engineering, 1993, 287 p.

[8] S. Hyun, and M.O. Robbins, "Elastic contact between rough surfaces: Effect of roughness at large and small wavelengths", Tribology Int., vol, 40, pp. 1413-1422, October-December 2007

[9] C.-D. Yeo, R.R. Katta, J. Lee, and A.A. Polycarpou, "Effect of asperity interactions on rough surface elastic contact behavior: Hard film on soft substrate", Tribology Int., vol. 43, pp. 1438-1448, August 2010

[10] Y. Xu, R.L Jackson, and D..B. Marghitu, "Statistical model of nearly complete elastic rough surface contact”, Int. J. Solids \& Structures, vol. 51, pp, 1075-1088, March 2014

[11] V. A. Yastrebov, G. Anciaux, and J.-F. Molinari, "From infinitesimal to full contact between rough surfaces: Evolution of the contact area", Int. J. Solids \& Structures, vol. 52, pp. 83-102, January 2015

[12] Y. Xu an R.L Jackson, "Statistical models of nearly complete elastic rough surface contact-comparison with numerical solutions", Tribology Int., vol. 105, pp. 274-291, January 2017

[13] P.M. Ogar, D.B. Gorokhov, and A.S. Kozhevnikov, "The density of gaps in the seal joint in elastic contact of microasperities", in Proc. 2nd International Conference on Modelling, Identification and Control (MIC 2015). Paris,. pp. 177-180, Aug. 2015

[14] V.P. Tihomirov, V.V. Poroshin, O.A. Gorlenko, and M.A. Izmerov, Tightness demountable fixed joints. Moscow: MSIU, 2014, 276 p. 